\title{
POWER-ASSOCIATIVE REGULAR REAL NORMED ALGEBRAS
}

\author{
EMANUEL STRZELECKI
}

(Received 3 August 1965)

\section{Introduction}

Definition 1. A real algebra $A$ is a real vector space in which an operation of multiplication is defined satisfying the following conditions:

(i) $\alpha(x y)=(\alpha x) y=x(\alpha y)$;

(ii) $(x+y) z=x z+y z$;

(iii) $x(y+z)=x y+x z$

for arbitrary $x, y, z \in A$ and any real number $\alpha$.

All algebras considered in this paper are supposed to have an identity element (denoted by $e$ ) but are not assumed associative or finite-dimensional.

Definition 2. A norm on $A$ is a real-valued function $x \rightarrow\|x\|$ on $A$ with the properties

(a) $\|x+y\| \leqq\|x\|+\|y\| ;$

(b) $\|\alpha x||=|x| \cdot\| x \|$;

(c) $\|x\| \neq 0$ if $x \neq 0$;

(d) $\|x y\| \leqq\|x\| \cdot\|y\|$;

(e) $\|e\|=1$.

Here $x, y$ are arbitrary elements belonging to $A$, and $\alpha$ is an arbitrary real number.

Definition 3. A prehilbert algebra is a normed algebra on which a positive definite inner product $(x, y)$ is defined, so that $\|x\|^{2}=(x, x)$.

Definition 4. A normed algebra $A$ is said to be absolute-valued if $\|x y\|=\|x\| \cdot\|y\|$ for all $x, y \in A$.

Definition 5. The unit element $e$ is said to be regular if there exists only one hyperplane of support to the unit sphere $S=\{x|||x| \mid=1\}$ at $e$. (See $\S 3$ for equivalent definitions and details.)

Definition 6. Let $A$-denote an algebra and $A(x)$ the subalgebra generated by the identity and an element $x . A$ is called 
(i) algebraic if $A(x)$ is finite dimensional;

(ii) one-sided alternative if for all $x, y \in A$ (a) $x^{2} y=x(x y)$ or $(b) x y^{2}=(x y) y$;

(iii) power-associative if $A(x)$ is associative for all $x$.

It was proved by $K$. Urbanik and F. B. Wright [9] that a real absolutevalued algebra with jdentity is isomorphic to the real numbers $(R)$, the complex numbers $(C)$, the quaternions $(Q)$ or the Cayley numbers $(D)$.

The author ([8], Theorem 2) has proved that an algebraic one-sided alternative real normed algebra with a regular unit element is isometrically isomorphic to $R, C, Q$ or $D$.

L. Ingelstam ([2], Theorem 3.1) obtained the same assertion for alternative real prehilbert algebras with identity. (The algebra was not assumed algebraic.) However, the assumption that the algebra is prehilbert algebra is more restrictive than the requirement of regularity of the unit element because in any prehilbert algebra every element $x$ with $\|x\|=1$ is regular.

We will show that every power associative real normed algebra with a regular unit element is an algebraic prehilbert algebra.

\section{Convex functionals and tangent functions}

Definition. A real-valued function $p(x)$ defined on all of the vector space $X$ is said to be convex if

(a) $p(x) \geqq 0$;

(b) $p(x)<+\infty$;

(c) $p(\alpha x)=\alpha p(x)$ for $\alpha \geqq 0$;

(d) $p(x+y) \leqq p(x)+p(y)$.

LEMMA 2.1. Let $p(x)$ be a convex functional defined on a linear space $\mathfrak{X}$. Then $[p(b+\gamma a)-p(a)] / \gamma$ is an increasing function of the positive variable $\gamma$ for arbitrary fixed $a, b$ in $\mathfrak{X}$. The limit

$$
\tau(b, a)=\lim _{\gamma \rightarrow 0+} \frac{1}{\gamma}[p(b+\gamma a)-p(b)]
$$

exists for each $a, b$ in $\mathfrak{X}$.

For proof of Lemma 2.1 as well as of Lemma 2.2 see [1]; V.9.

Definition. If $p(x)$ is a convex functional, then the function $\tau(x, y)$ defined for $x, y \in \mathfrak{X}$ by

$$
\tau(x, y)=\lim _{\gamma \rightarrow 0+} \frac{1}{\gamma}[p(x+\gamma y)-p(x)]
$$

is called the tangent function of $p(x)$. 
LEMMA 2.2. Let $\tau(x, y)$ be the tangent function of a convex functional $p(x)$ defined in a linear space $\mathfrak{X}$. Then

(a) $\tau(x, y) \leqq p(y)$;

(b) $\tau\left(x, y_{1}+y_{2}\right) \leqq \tau\left(x, y_{1}\right)+\tau\left(x, y_{2}\right)$;

(c) $\tau(x, \alpha y)=\alpha \tau(x, y)$ for $\alpha \geqq 0$;

(d) $-\tau(x,-y) \leqq \tau(x, y)$;

(e) $\tau(x, \alpha x)=\alpha p(x)$.

LEMмa 2.3. Let $a, b$ be fixed vectors in a linear space $\mathfrak{X}$ and let $\tau(x, y)$ be the tangent function of a convex functional $p(x)$ defined in $\mathfrak{X}$. Then the function $\varphi(\xi)=\tau(b+\xi a, a)$ does not decrease as $\xi \rightarrow+\infty$.

Proof. We have to show that $\varphi\left(\xi_{2}\right)-\varphi\left(\xi_{1}\right) \geqq 0$ when $\xi_{2}>\xi_{1}$. By the definition of $\tau(x, y)$, we have

$$
\begin{aligned}
\varphi\left(\xi_{2}\right)-\varphi\left(\xi_{1}\right)= & \tau\left(b+\xi_{2} a, a\right)-\tau\left(b+\xi_{1} a, a\right) \\
= & \lim _{\gamma \rightarrow 0+} \frac{1}{\gamma}\left[p\left(b+\xi_{2} a+\gamma a\right)-p\left(b+\xi_{2} a\right)\right] \\
& -\lim _{\gamma \rightarrow 0+} \frac{1}{\gamma}\left[p\left(b+\xi_{1} a+\gamma a\right)-p\left(b+\xi_{1} a\right)\right] \\
= & \lim _{\gamma \rightarrow 0+} \frac{1}{\gamma}\left[p\left(b+\xi_{1} a\right)+p\left(b+\xi_{2} a+\gamma a\right)-p\left(b+\xi_{2} a\right)-p\left(b+\xi_{1} a+\gamma a\right)\right] .
\end{aligned}
$$

Thus, it is sufficient to verify that the expression in the brackets is nonnegative for $\xi_{2}>\xi_{1}$. Since

$$
b+\xi_{2} a=\left(\xi_{2}-\xi_{1}+\gamma\right)^{-1}\left[\gamma\left(b+\xi_{1} a\right)+\left(\xi_{2}-\xi_{1}\right)\left(b+\xi_{2} a+\gamma a\right)\right]
$$

and $\xi_{2}-\xi_{1}>0, \gamma>0$, by the properties (c) and (d) of a convex functional, we get the inequality

(1) $p\left(b+\xi_{2} a\right) \leqq\left(\xi_{2}-\xi_{1}+\gamma\right)^{-1}\left[\gamma p\left(b+\xi_{1} a\right)+\left(\xi_{2}-\xi_{1}\right) p\left(b+\xi_{2} a+\gamma a\right)\right]$.

By the same reasoning

(2) $p\left(b+\xi_{1} a+\gamma a\right) \leqq\left(\xi_{2}-\xi_{1}+\gamma\right)^{-1}\left[\left(\xi_{2}-\xi_{1}\right) p\left(b+\xi_{1} a\right)+\gamma p\left(b+\xi_{2} a+\gamma a\right)\right]$.

Adding inequalities (1) and (2) we obtain the needed result. //

Lemma 2.4. If $a, b$ are fixed vectors in a linear space $\mathfrak{X}$ and $\tau(x, y)$ is the tangent function of a convex functional $p(x)$, then

$$
\lim _{\xi \rightarrow \infty} \tau(b+\xi a, a)=p(a)
$$

Proof. By 2.2 (a) and 2.3 the limit exists and

$$
\lim _{\xi \rightarrow \infty} \tau(b+\xi a, a) \leqq p(a) .
$$


On the other hand, in view of the definition of a convex functional $p(x)$ the following inequalities hold for $\gamma>0, \xi>0$ :

$$
\begin{aligned}
\frac{1}{\gamma}[p(b+\xi a+\gamma a)-p(b+\xi a)] \\
\quad \geqq \frac{1}{\gamma}\left[p\left(b+\frac{\gamma}{\xi} b+\xi a+\gamma a\right)-p(b+\xi a)-\frac{\gamma}{\xi} p(b)\right] \\
\quad=\frac{1}{\gamma}\left[(\xi+\gamma) p\left(\frac{b}{\xi}+a\right)-\xi p\left(\frac{b}{\xi}+a\right)\right]-\frac{1}{\xi} p(b) \\
=p\left(\frac{b}{\xi}+a\right)-\frac{1}{\xi} p(b) \geqq p(a)-\frac{1}{\xi} p(b)-\frac{1}{\xi}(-b) .
\end{aligned}
$$

Hence

$$
\lim _{\xi \rightarrow \infty} \tau(b+\xi a, a) \geqq p(a) .
$$

The comparison of inequalities (3) and (4) yields

$$
\lim _{\xi \rightarrow \infty} \tau(b+\xi a, a)=p(a) . / 1
$$

Lemma 2.5. Let $\varphi(\xi)=\tau(b+\xi a, a)$ denote the function of $\xi$ mentioned in Lemmas 2.3 and 2.4. Then for every $v$ there exists $\xi_{0}>v$ such that

$$
\tau\left(b+\left(\xi_{0}+\delta\right) a, a\right)-\tau\left(b+\xi_{0} a, a\right)=O(\delta)
$$

where $O(\delta)$ denotes a function of $\delta$ with the property

$$
\varlimsup_{\delta \rightarrow 0}\left|\frac{O(\delta)}{\delta}\right|<+\infty
$$

Proof. The assertion of our Lemma follows immediately from Lemma 2.3 inasmuch as a function which does not decrease has a finite derivative almost everywhere (see e.g. [6], Ch. VIII, § 2).//

Lemma 2.6. Let $p_{1}(x)$ and $p_{2}(x)$ be two convex functionals with $p_{2}(x)$ $\leqq p_{1}(x)$ for all $x \in \mathfrak{X}$. Let $\tau_{1}(x, y)$ and $\tau_{2}(x, y)$ be the tangent functions of $p_{1}(x)$ and $p_{2}(x)$ respectively. If two vectors $b$ and $q$ fulfil conditions

(a) $\tau_{1}(b,-q)=\tau_{1}(b, q)=0$,

(b) $p_{\mathbf{2}}(b)=p_{\mathbf{1}}(b)$,

then $\tau_{2}(b,-q)=\tau_{2}(b, q)=0$.

Proof. By the definition of a tangent function 


$$
\begin{aligned}
\tau_{2}(b, q) & =\lim _{\gamma \rightarrow 0+} \frac{1}{\gamma}\left[p_{2}(b+\gamma q)-p_{2}(b)\right] \\
& \leqq \lim _{\gamma \rightarrow 0+} \frac{1}{\gamma}\left[p_{1}(b+\gamma q)-p_{1}(b)\right]=\tau_{1}(b, q) .
\end{aligned}
$$

For the same reason

$$
\tau_{2}(b,-q) \leqq \tau_{1}(b,-q) .
$$

Therefore, by Lemma 2.2 (d) we get

$$
0=-\tau_{1}(b,-q) \leqq-\tau_{2}(b,-q) \leqq \tau_{2}(b, q) \leqq \tau_{1}(b, q)=0 .
$$

Thus, Lemma 2.6 is proved. //

\section{Regular bounding point of a convex set}

Definition. A point $a$ belonging to a subset $S$ of the linear space $\mathfrak{X}$ is called an internal point of $S$ if, for each $x \in \mathfrak{X}$, there exists an $\varepsilon>0$ such that $a+\gamma x \in S$ for $|\gamma| \leqq \varepsilon$. A point $b \in \mathfrak{X}$ is called a bounding point of $S$ if $b$ is an internal point of neither $S$ nor the complement of $S$.

Definition. If $S$ is a subset of a linear space $\mathfrak{X}$ and $b$ is a bounding point of $S$, a linear functional $f$ is said to be of support to $S$ at $b$ iff $f(x) \leqq f(b)$ for all $x \in S$. In this case the set $H=\{x \mid f(x)=f(b)\}$ is called a hyperplane of support to $S$ at the point $b$.

Definition. A bounding point $b$ of a subset $S$ of a linear space $\mathfrak{X}$ is said to be regular if there exists one and only one hyperplane $H$ of support to $S$ at the point $b$.

Definition. A set $K$ belonging to a vector space $\mathfrak{X}$ is convex if $x, y \in K$, and $0 \leqq \alpha \leqq 1$, imply $\alpha x+(1-\alpha) y \in K$.

LEMмA 3.1. If $p(x)$ is a convex functional and $\gamma>0$, then the set $K=$ $\{x \mid p(x) \leqq \gamma\}$ is a convex set containing the origin as an internal point. The set of bounding points is characterized by the condition $p(x)=\gamma$.

For proof of this Lemma see for example [5], $\S 1.8$.

DEFINITION. A vector $r$ is said to be a regular point of a convex functional $p(x)$ if

(a) $p(r)>0$,

(b) $r$ is a regular bounding point of the set $K=\{x \mid p(x) \leqq p(r)\}$.

THEOREM 3.2. Let $\mathfrak{X}$ be a linear space and $\tau(x, y)$ a tangent function of a convex functional $p(x)$. Then if $p(b)>0$, a linear functional $f(x)$ with 
$f(b)=p(b)$ is a functional of support to $K=\{x \mid p(x) \leqq p(b)\}$ at $b$ if and only if $-\tau(b,-y) \leqq f(y) \leqq \tau(b, y)$ for all $y \in \mathfrak{X}$.

Conversely, if $p(b)>0$, and if $\alpha$ is any real number and $a$ any point in $\mathfrak{X}$ for which

$$
-\tau(b,-a) \leqq \alpha \leqq \tau(b, a)
$$

then there is a functional of support to $K=\{x \mid p(x) \leqq p(b)\}$ at $b$ with $f(b)$ $=p(b), f(a)=\alpha, f(x) \leqq p(x)$ for all $x \in \mathfrak{X}$.

Notes. 1. The inequality $f(x) \leqq p(x)$ follows from (a) of Lemma 2.2. 2. For the case $p(b)=1$ the theorem is proved in [1], V.9.4. We get the proof of our case putting $p_{1}(x)=p(x) / p(b)$.

Lemma 3.3. Let $\tau(x, y)$ be the tangent function of a convex functional $p(x)$. If two vectors $b$ and $q$ fulfil the conditions $p(b)>0, \tau(b, q)=0$, then

(1) there exists a linear functional $f(x)$ such that:

(a) $f(b)=p(b)$;

(b) $f(q)=0$;

(c) $f(x) \leqq p(x)$ for all $x \in \mathfrak{X}$.

(2) $p(b+\xi q) \geqq p(b)$ for all $\xi$.

PRoof. The first assertion follows immediately from Theorem 3.2.

Now let $f(x)$ be the linear functional for which conditions (a), (b), (c) hold. Then

$$
p(b+\xi q) \geqq f(b+\xi q)=f(b)=p(b) . / /
$$

The next lemma is a direct consequence of Theorem 3.2.

LEMMA 3.4. Let $\tau(x, y)$ be the tangent function of a convex functional $p(x)$. If $p(b)>0$ then there exists a unique linear functional $f(x)$ of support to the convex set $K=\{x \mid p(x) \leqq p(b)\}$ at the point $b$ with $f(b)=p(b)$ if and only if

$$
-\tau(b,-y)=\tau(b, y) \text { for all } y \in \mathfrak{X} \text {. }
$$

In this connection

$$
f(x)=\tau(b, x) .
$$

Lemma 3.4 implies the following propositions:

THEOREM 3.5. Let $\tau(x, y)$ be the tangent function of a convex functional $p(a) . A$ vector $r$ is a regular point of the convex functional $p(x)$ if and only if

(a) $p(r)>0$;

(b) $-\tau(r,-y)=\tau(r, y)$ for all $y \in \mathfrak{X}$.

LEMMA 3.6. Let $\tau(x, y)$ be the tangent function of a convex functional 
$p(x)$. If $r$ is a regular point of the convex functional $p(x)$, then $\tau(r, x)$ is a linear functional with $\tau(r, r)=p(r)>0$.

LEMMA 3.7. Let $r$ be a regular point of a convex functional $p(x)$. If a is an arbitrary vector in $\mathfrak{X}$, then there exists a vector $q=a+\lambda r$ for which

$$
\tau(r,-q)=\tau(r, q)=0 \text {. }
$$

Proof. By the definition of a regular point of a convex functional $p(x)$, $p(r)>0$. Then, by Lemma 3.6, $\tau(r, r)=p(r) \neq 0$. Now it is easy to verify that

$$
q=a-\frac{\tau(r, a)}{\tau(r, r)} r
$$

is the needed vector. In fact, by Lemma 3.6, the functional $f(x)=\tau(r, x)$ is linear when $r$ is a regular point of $p(x)$. Therefore

$$
\tau(r, q)=\tau\left(r, a-\frac{\tau(r, a)}{\tau(r, r)} r\right)=\tau(r, a)-\frac{\tau(r, a)}{\tau(r, r)} \tau(r, r)=0 .
$$

Since $r$ is regular we have also

$$
-\tau(r,-q)=\tau(r, q)=0 . / 1
$$

\section{Geometric properties of real normed algebras}

Let $A$ be a real normed algebra with a unit element $e$. Obviously the norm $\|x\|$ is a convex functional in $A$. In the following sections $\tau(x, y)$ will denote the tangent function of the convex functional $p(x)=\|x\|$.

LEMMA 4.1. Let $A$ be a real normed algebra. If $q$ is a vector for which $\tau(e,-q)=\tau(e, q)=0$, then $\tau(x,-q x)=\tau(x, q x)=0$ for every $x \in \mathfrak{X}$.

Proof. Since $\|y x\| \leqq\|y\| \cdot\|x\|$ for every $y, x \in A$, we have:

$$
\begin{gathered}
\|x+\gamma q x\|=\|(e+\gamma q) x\| \leqq\|e+\gamma q\| \cdot\|x\|, \\
\|x+\gamma(-q x)\|=\|(e+\gamma(-q)) x\| \leqq\|e+\gamma(-q)\| \cdot\|x\| .
\end{gathered}
$$

Consequently, for $\gamma>0$

$$
\begin{aligned}
& \frac{1}{\gamma}(\|x+\gamma q x\|-\|x\|) \leqq \frac{1}{\gamma}(\|e+\gamma q\|-1) \cdot\|x\|, \\
& \frac{1}{\gamma}(\|x+\gamma(-q x)\|-\|x\|) \leqq \frac{1}{\gamma}(\|e+\gamma(-q)\|-1) \cdot\|x\| .
\end{aligned}
$$

Passing to a limit as $\gamma \rightarrow 0+$ in those inequalities in view of $\tau(e,-q)=$ $\tau(e, q)=0$, we obtain 


$$
\begin{gathered}
\tau(x, q x) \leqq \tau(e, q) \cdot\|x\|=0, \\
\tau(x,-q x) \leqq \tau(e,-q) \cdot\|x\|=0 .
\end{gathered}
$$

Consequently, by $2.4(\mathrm{~d})$,

which implies

$$
0 \leqq-\tau(x,-q x) \leqq \tau(x, q x) \leqq 0,
$$

$$
\tau(x,-q x)=\tau(x, q x)=0 . / /
$$

LEMMA 4.2. Let $A$ be a real normed algebra; if $q$ is a vector for which $\tau(e,-q)=\tau(e, q)=0$ then

$$
1 \leqq\|e+\xi q\| \leqq\left\|e-\xi^{2} q^{2}\right\|
$$

Proof. Since $\|e\|=1$, the inequality $\|e+\xi q\| \geqq 1$ follows immediately from Lemma 3.3(2) if we put $p(x)=\|x\|, b=e$.

By the previous Lemma, $\tau(x,-q x)=\tau(x, q x)=0$ for every $x \in \mathfrak{X}$, for $\tau(e,-q)=\tau(e, q)=0$. Replacing here $x$ by $e+\xi q$, we obtain $\tau\left(e+\xi q, q+\xi q^{2}\right)=0$. Then, by Lemma 3.3(1), we conclude that there exists a linear functional $f(x)$ such that:

(a) $f(e+\xi q)=\|e+\xi q\|$,

(b) $f\left(q+\xi q^{2}\right)=0$,

(c) $f(x) \leqq\|x\|$ for all $x \in \mathfrak{X}$.

From (b) we obtain

$$
f(\xi q)=-f\left(\xi^{2} q^{2}\right)
$$

Therefore from (a) and (c) we get

$$
\|e+\xi q\|=f(e)+f(\xi q)=f(e)-f\left(\xi^{2} q^{2}\right)=f\left(e-\xi^{2} q^{2}\right) \leqq\left\|e-\xi^{2} q^{2}\right\| \cdot \|
$$

LEMMA 4.3. Let $A$ be a real normed algebra. If $q$ is a vector for which $\tau(e,-q)=\tau(e, q)=0$ then

$$
0 \leqq\|e+\xi q\|-1 \leqq \xi^{2}\left\|q^{2}\right\| \text { for every number } \xi \text {. }
$$

Proof. By the previous lemma we obtain

$$
0 \leqq\|e+\xi q\|-1 \leqq\left\|e-\xi^{2} q^{2}\right\|-1 \leqq\|e\|+\left\|\xi^{2} q^{2}\right\|-1=\xi^{2}\left\|q^{2}\right\| \cdot \|
$$

LEMMA 4.4. Let $A$ be a real normed algebra. If $q$ is a vector for which $\tau(e,-q)=\tau(e, q)=0$ and $\tau\left(e,-q^{2}\right)=\left(e, q^{2}\right)=0$ then

$$
0 \leqq\|e+\xi q\|-1 \leqq \xi^{4}\left\|\left(q^{2}\right)^{2}\right\| \text {. }
$$

Proof. Since $\tau\left(e,-q^{2}\right)=\tau\left(e, q^{2}\right)=0$, we can apply the previous lemma to the expression $\left\|e-\xi^{2} q^{2}\right\|-1$. Therefore, in view of Lemma 4.2 we obtain

$$
0 \leqq\|e+\xi q\|-1 \leqq\left\|e-\xi^{2} q^{2}\right\|-1 \leqq \xi^{4}\left\|\left(q^{2}\right)^{2}\right\| . \|
$$


LEMMA 4.5. Let $A$ be a real normed algebra. If $q$ is a vector for which $\|q\|>0, \tau(e,-q)=\tau(e, q)=0$, then either $\tau\left(e,-q^{2}\right) \neq 0$ or $\tau\left(e, q^{2}\right) \neq 0$.

Proof. Let us take $\xi>0, \eta>0, \delta>0$. Since $\|x y\| \leqq\|x\| \cdot\|y\|$ for all $x, y \in A$, we have

$$
\begin{gathered}
\left\|e+(\xi+\eta) q+\xi \eta q^{2}\right\| \leqq\|e+\xi q\| \cdot\|e+\eta q\|, \\
\| e+\left(\left(\xi+\delta-\frac{\xi \eta}{\xi+\delta}\right) q-\xi \eta q^{2}\|\leqq\| e+(\xi+\delta) q\|\cdot\| e-\frac{\xi \eta}{\xi+\delta} q \| .\right.
\end{gathered}
$$

We know that $\|x+y\| \leqq\|x\|+\|y\|$ for all $x, y \in A$. Therefore adding these inequalities we get

$$
\begin{aligned}
2\left\|e+\xi q+\frac{\delta}{2}\left(1+\frac{\eta}{\xi+\delta}\right) q\right\| \leqq & \|e+\xi q\| \cdot\|e+\eta q\| \\
& +\|e+(\xi+\delta) q\|\left\|e-\frac{\xi \eta}{\xi+\eta} q\right\| .
\end{aligned}
$$

By Lemma 2.1, for $\gamma>0$ we have

$$
\tau(b, a)=\lim _{\gamma \rightarrow 0+}(\|b+\gamma a\|-\|b\|) / \gamma \leqq(\|b+\gamma a\|-\|b\|) / \gamma
$$

or

(2)

$$
\gamma \tau(b, a) \leqq\|b+\gamma a\|-\|b\| .
$$

Substituting here $b=e+\xi q, a=q, \gamma=\delta(1+\eta /(\xi+\delta)) / 2$ we obtain

(3) $\delta\left(1+\frac{\eta}{\xi+\delta}\right) \tau(e+\xi q, q) \leqq 2\left\|e+\xi q+\frac{\delta}{2}\left(1+\frac{\eta}{\xi+\delta}\right) q\right\|-2\|e+\xi q\|$.

Inequalities (1) and (3) imply

$$
\begin{aligned}
& \delta\left(1+\frac{\eta}{\xi+\delta}\right) \tau(e+\xi q, q) \\
& \quad \leqq\|e+\xi q\|(\|e+\eta q\|-1)+\|e+(\xi+\delta) q\| \cdot\left\|e-\frac{\xi \eta}{\xi+\delta} q\right\|-\|e+\xi q\| .
\end{aligned}
$$

Substituting in (2) $b=e+(\xi+\delta) q, a=-q, \gamma=\delta$ we obtain

Therefore

$$
\delta \tau(e+(\xi+\delta) q,-q) \leqq\|e+\xi q\|-\|e+(\xi+\delta) q\| .
$$

$$
\|e+(\xi+\delta) q\| \leqq\|e+\xi q\|-\delta \tau(e+(\xi+\delta) q,-q) .
$$

Consequently, by Lemma 2.2 (d), the inequality

$$
\|e+(\xi+\delta) q\| \leqq\|e+\xi q\|+\delta \cdot \tau(e+(\xi+\delta) q, q)
$$

holds. 
Since, by the assumptions, $\|q\|>0$, in view of Lemma 2.4 there exists a positive number $v$ such that $\tau(e+v q, q)>0$. Let us choose $\xi_{0}>v$ according to Lemma 2.5. Then for $\xi_{0}$ inequality (5) yields

$$
\left\|e+\left(\xi_{0}+\delta\right) q\right\| \leqq\left\|e+\xi_{0} q\right\|+\delta \tau\left(e+\xi_{0} q, q\right)+\delta \cdot O(\delta) .
$$

Inequalities (4) and (6) imply

$$
\begin{aligned}
& \frac{\delta \eta}{\xi_{0}+\delta} \tau\left(e+\xi_{0} q, q\right) \leqq\left\|e+\xi_{0} q\right\|\left(\|e+\eta q\|-1+\left\|e-\frac{\xi_{0} \eta}{\xi_{0}+\delta}\right\|-1\right) \\
& \quad+\delta \tau\left(e+\xi_{0} q, q\right)\left(\left\|e-\frac{\xi_{0} \eta}{\xi_{0}+\delta} q\right\|-1\right)+\delta \cdot O(\delta) \cdot\left\|e-\frac{\xi_{0} \eta}{\xi_{0}+\delta} q\right\| .
\end{aligned}
$$

Now, let us suppose that we have simultaneously $\tau\left(e,-q^{2}\right)=\tau\left(e, q^{2}\right)$ $=0$. Then we can apply Lemma 4.4 to the last inequality obtaining

where

$$
\frac{\delta \eta}{\xi_{0}+\delta} \tau\left(e+\xi_{0} q, q\right) \leqq \eta^{4} \cdot N(\delta)+\delta \cdot O(\delta) \cdot\left\|e-\frac{\xi_{0} \eta}{\xi_{0}+\delta} q\right\| .
$$

$$
N(\delta)=\left\|\left(q^{2}\right)^{2}\right\| \cdot\left\{\left\|e+\xi_{0} q\right\|\left[1+\left(\frac{\xi_{0}}{\xi_{0}+\delta}\right)^{4}\right]+\delta\left(\frac{\xi_{0}}{\xi_{0}+\delta}\right)^{4} \tau\left(e+\xi_{0} q, q\right)\right\} .
$$

Let us replace $\eta$ by $\delta^{2}$. Then dividing both sides of the last inequality by $\delta$ we obtain

$$
\left(\xi_{0}+\delta\right)^{-1} \tau\left(e+\xi_{0} q, q\right) \leqq \delta \cdot N(\delta)+\delta^{\frac{1}{3}} \cdot \frac{O(\delta)}{\delta} \cdot\left\|e-\frac{\xi_{0} \eta}{\xi_{0}+\delta} q\right\| .
$$

Tending to a limit as $\delta \rightarrow 0+$, in view of $\overline{\lim }_{\delta \rightarrow 0}|O(\delta) / \delta|<\infty$, we get

$$
\xi_{0}^{-1} \tau\left(e+\xi_{0} q, q\right) \leqq 0,
$$

which is impossible because $\xi_{0}>0$ and by Lemma 2.3

$$
\tau\left(e+\xi_{0} q, q\right) \geqq \tau(e+v q, q)>0 .
$$

Thus, Lemma 4.5 is proved. //

\section{Real commutative Banach algebras}

Definition. A real commutative Banach algebra $B$ is a commutative, associative real normed algebra with unit element $e$ whose vector space is a complete normed space.

Definition. A set $I$ of elements of a commutative Banach algebra $B$ is called an ideal if it has the following properties: 
(a) if $x \in I$ and $y \in I$, then $x+y \in F$;

(b) if $x \in I$, then $z x \in I$ for all $z \in B$.

An ideal $I$ of a Banach algebra is called a proper ideal if, in addition,

(c) $I \neq B$.

Note. The unit element $e$ does not belong to any proper ideal. For, if $e \in I$ then, by 2 (b), $z=z e \in I$ where $z$ is an arbitrary element of $B$. Therefore $e \in I$ implies $I=B$.

Definition. A maximal ideal is a proper ideal that is not contained in any other proper ideal.

Definition. The radical $B$ of a commutative Banach algebra is the intersection of all maximal ideals.

Obviously the radical of a Banach algebra is a proper ideal.

THEOREM 5.1. Given a real commutative Banach algebra $B$ there exists a homomorphic mapping $x \rightarrow \hat{x}$ of $B$ onto an algebra $\hat{B}$ of complex functions defined in the space $\phi$ of all homomorphisms of $B$ onto the complex field. In this connection the following properties hold:

(a) If $x=x_{1}+x_{2}$, then $\hat{x}(\varphi)=\hat{x}_{1}(\varphi)+\hat{x}_{2}(\varphi)$;

(b) If $x=x_{1} \cdot x_{2}$, then $\hat{x}(\varphi)=\hat{x}_{1}(\varphi) \cdot \hat{x}_{2}(\varphi)$;

(c) If $x_{2}=\lambda x_{1}$, then $\hat{x}_{2}(\varphi)=\lambda \hat{x}_{1}(\varphi)$;

(d) $\hat{e}(\varphi) \equiv 1, \quad$ where $e$ is the unit element of $B$;

(e) $\hat{x}(\varphi) \equiv 0 \Leftrightarrow x$ belongs to the radical of the algebra $B$;

(f) $|\hat{x}(\varphi)| \leqq\|x\|$.

For the proof of this theorem see e.g. [3], p. 245-247 or [7], Ch. III § 1 . The property (f) follows from the inequality:

$$
|\hat{x}(\varphi)| \leqq \lim _{n \rightarrow \infty}\left\|x^{n}||^{1 / n} \leqq \lim _{n \rightarrow \infty}\right\| x\left\|^{n / n}=\right\| x \| .
$$

Definition. The complex functions $\hat{x}(\varphi)$ with the properties (a)-(f) will be called Gelfand functions. The homomorphic mapping $x \rightarrow \hat{x}(\varphi)$ is called Gelfand's representation of the algebra $B$.

\section{Regular real power-associative normed algebras.}

Definition. A real normed algebra $A$ is said to be regular if the unit element $e$ is a regular point of the convex functional $p(x)=\|x\|$.

Note. It is easy to verify that a real normed algebra $A$ is regular iff there exists just one linear functional $f$ on $A$ satisfying $f(e)=1$ and $f(x) \leqq\|x\|$. 
LEMMA 6.1. If $A$ is a normed algebra and $\tau(x, y)$ is the tangent function of a convex functional $p(x)=\|x\|$ then, for every fixed $a, \tau(a, y)$ is a continuous function of $y$.

Proof. This lemma is a consequence of the inequality

$$
\mid \tau\left(x, y_{2}\right)-\tau\left(x, y_{1}\right) \leqq\left\|y_{2}-y_{1}\right\|
$$

which follows from Lemmas 2.2 (a) and $2.2(\mathrm{~b})$. //

LeMma 6.2. Let $b$ be a nonzero vector of a real normed algebra $A$. If the equality $-\tau(b,-z)=\tau(b, z)$ holds for every vector $z$ belonging to a set $Z$ that is dense in $A$, then $b$ is a regular point of $p(x)=\|x\|$.

This lemma follows immediately from 6.1 and 3.5 , since $\|b\| \neq 0$ for $b \neq 0$.

Lemma 6.3. Every non-complete regular real normed algebra $A$ can be embedded in a complete regular normed algebra $A^{\prime}$. $A^{\prime}$ can be chosen in such a way that $A$ will be dense in $A^{\prime}$.

For proof of Lemma 6.3 see $[5], \S 4.1$ and $\S 9.1$. The regularity of $A^{\prime}$ follows from the previous lemma. It is easy to verify that if $A$ is power-associative then $A^{\prime}$ has the same property.

THEOREM 6.4. Every regular power-associative real normed algebra is algebraic.

Proof. If $A$ is one dimensional we have nothing to prove. Otherwise there exists an element $a \in A$ such that $a$ and $e$ are linearly independent. Since $e$ is a regular point of the convex functional $p(x)=\|x\|$, by Lemma 3.7, there exists a vector $q=a+\lambda . e$ for which $\tau(e, q)=0$, where $\tau(x, y)$ is the tangent function of $p(x)=\|x\| .\|q\| \neq 0$, for $q \neq 0, q$ being a vector linearly independent of the unit element $e$. Let us consider the subalgebra $A(q)$ generated by identity and the element $q$. In view of Theorem 3.5, $A(q)$ is regular being a subalgebra with identity of a regular algebra. If $A(q)$ is not complete, let $A^{\prime}(q)$ denote the completion of $A(q)$. By Lemma $6.3, A^{\prime}(q)$ is regular. Since $A$ is power-associative, $A^{\prime}(q)$ is a real commutative Banach algebra. Therefore we can use the Gelfand representation of $A^{\prime}(q)$. Let us define in $A^{\prime}(q)$ a functional $p_{2}(x)$ by means of the formula

$$
p_{2}(x)=\sup _{\varphi \in \phi}|\hat{x}(\varphi)| \text {. }
$$

Obviously $p_{2}(x)$ is a convex functional. From $5.1(\mathrm{~d})$ and $5.1(\mathrm{f})$ it follows that

$$
\begin{aligned}
& p_{2}(e)=\sup _{\varphi \in \phi}|\hat{e}(\varphi)|=1=\|e\|, \\
& p_{2}(x) \leqq\|x\| \text { for every } x \in A^{\prime}(q) .
\end{aligned}
$$


Therefore, by Lemma 2.6, $\tau_{2}(e,-q)=\tau_{2}(e, q)=0$ since, by Lemma 3.4, $\tau(e,-q)=\tau(e, q)=0$. (Here $\tau_{2}(x, y)$ denotes the tangent function of the convex functional $\left.p_{2}(x)\right)$.

We shall prove that $\operatorname{Re} \hat{q}(\varphi)=0$. In fact, let $\hat{q}\left(\varphi_{0}\right)=\alpha+\beta i$. By Theorem 5.1, for $\gamma>0$, we get

$$
\begin{aligned}
{\left[p_{2}(e+\gamma q)-p_{2}(e)\right] / \gamma } & =\left[\sup _{\phi \in \phi}|\widehat{e+\gamma q}(\varphi)|-1\right] / \gamma \\
& =\left[\sup _{\phi \in \phi}|\hat{e}(\varphi)+\gamma \hat{q}(\varphi)|-1\right] / \gamma \geqq[|1+\gamma \alpha+\gamma \beta i|-1] / \gamma \\
& =\left[\left(1+2 \gamma \alpha+\gamma^{2}\left(\alpha^{2}+\beta^{2}\right)\right)^{\frac{1}{2}}-1\right] / \gamma .
\end{aligned}
$$

Therefore

$$
0=\tau_{2}(e, q)=\lim _{\gamma \rightarrow 0+}\left[p_{2}(e+\gamma q)-p_{2}(e)\right] / \gamma \geqq \alpha .
$$

For the same reason

$$
0=\tau_{2}(e,-q) \geqq-\alpha .
$$

The two inequalities imply $\alpha=0$.

Let us consider now the element $q^{2}$ and its Gelfand function $\widehat{q}^{2}(p)$. In view of Theorem 5.1 (b) $\widehat{q^{2}}(\varphi)=[\hat{q}(\varphi)]^{2}$. Hence $\operatorname{Re} \hat{q}(\varphi)=0$ implies $\operatorname{Im} \widehat{q^{2}}(\varphi)=0$.

Since $e$ is a regular point of $p(x)=\|x\|_{1}$, by Lemma 3.7, there exists a number $\mu$ such that for a vector $q_{1}=q^{2}+\mu e$ we get the equalities $\tau\left(e,-\left(q^{2}+\mu e\right)\right)=\tau\left(e, q^{2}+\mu e\right)=0$. We note that $\mu \neq 0$. In fact, if $\mu=0$, we have simultaneously $\|q\| \neq 0, \tau(e,-q)=\tau(e, q)=0, \tau\left(e,-q^{2}\right)=$ $\tau\left(e, q^{2}\right)=0$ which contradicts Lemma 4.5 . Now applying the same argument as in the case of the function $\hat{q}(\varphi)$ we conclude that $\operatorname{Re} \hat{q}_{1}(\varphi)=0$. But $\operatorname{Im} \hat{q}_{1}(\varphi)=\operatorname{Im} \widehat{q^{2}+\mu e}(\varphi)=\operatorname{Im} \widehat{q^{2}}(\varphi)+\operatorname{Im}, \mu=0$, since $\operatorname{Im} \widehat{q^{2}}(\varphi)=0$ and $\mu$ is a real number. Therefore

$$
\hat{q}_{1}(\varphi)=0 .
$$

and consequently, by Theorem $5.1(\mathrm{e}), q_{1}=q^{2}+$, $u$ e belongs to the radical of the subalgebra $A^{\prime}(q)$.

We shall prove that

$$
q_{1}=q^{2}+\mu e=0 .
$$

Let us suppose that $q_{1} \neq 0$. Then $\left\|q_{1}\right\| \neq 0$ and as was mentioned before $\tau\left(e,-q_{1}\right)=\tau\left(e, q_{1}\right)=0$. Therefore, by the previous considerations about the vector $q$, there exists a nonzero number $\chi$ such that the vector $q_{2}=q_{1}^{2}+\chi e$ belongs to the radical of the subalgebra $A^{\prime}(q)$. But $q_{1}^{2}$ belongs to the radical since $q_{1}$ has this property. Then $e=1 / \chi\left(q_{2}-q_{1}^{2}\right)$ belongs to the radical which is a contradiction inasmuch as $e$ does not belong to any proper ideal of $A^{\prime}(q)$. 
Thus we have proved that there exists a real number $\mu$ such that

$$
q^{2}+\mu e=0 .
$$

Taking into account that $q=a+\lambda e$ we conclude that the algebra $A(a)$ is two-dimensional. //

COROLLARY 6.5. If $A$ is a regular power-associative real normed algebra and $a$ is an element that is linearly independent of the unit element $e$, then the subalgebra $A(a)$ is isomorphic to the complex field $C$.

Proof. Since $\operatorname{Re} \hat{q}(\varphi)=0,(\hat{q}(\varphi))^{2} \leqq 0$. Therefore the equality $\hat{q}_{1}(\varphi)=$ $(\hat{q}(\varphi))^{2}+\mu=0$ implies $\mu>0$, for $\mu \neq 0$. Consequently, from $q^{2}+\mu e=0$ it follows that the element $i=\mu^{-\frac{1}{2}} q$ fulfils the equality $i^{2}=-e . / /$

COROLLARY 6.6. A regular power-associative real normed algebra contains no idempotents other than zero and the unit element, and no non-zero nilpotents.

Proof. The assertions follow from the fact that in the complex field the equality $x^{2}=x$ has only two solutions: $x_{1}=0$ and $x_{2}=1$ and the equality $x^{2}=0$ is fulfilled only for $x=0$. //

Another proof of this corollary is presented in [8], Lemmas 6 and 8.

THEOREM 6.7. In a regular power-associative real normed algebra $A$ the norm $\|||$ is generated by an inner product, i.e. $A$ is a real prehilbert algebra.

Proof. According to [4] it is sufficient to show that every twodimensional subspace of $A$ is a Euclidean space. Let $a$ be an element of $A$ that is not a scalar multiple of $e$. By Corollary 6.5, $A(a)$ is isomorphic to the complex field, i.e. there exists an element $i_{a} \in A(a)$ such that $i_{a}^{2}=-e$. Obviously $e$ and $i_{a}$ are linearly independent and consequently form a basis of the twodimensional space $A(a)$. Since $e$ is regular, the norm of an element $\alpha e+\beta i$ can be expressed by the formula

(see [8], Lemma 10).

$$
\left\|\alpha e+\beta i_{a}\right\|=\left(\alpha^{2}+\beta^{2}\right)^{2} ;
$$

Now, let us define in $A(a)$ the inner product of two elements $\alpha_{1} e+\beta_{1} i_{a}$ and $\alpha_{2} e+\beta_{2} i_{a}$ as following

$$
\left(\alpha_{1} e+\beta_{1} i_{a}, \alpha_{2} e+\beta_{2} i_{a}\right)=\left(\alpha_{1} \alpha_{2}+\beta_{1} \beta_{2}\right) .
$$

Then we obtain

$$
\left\|\alpha e+\beta i_{a}\right\|=\left(\alpha e+\beta i_{a}, \alpha e+\beta i_{a}\right)^{\frac{1}{2}}
$$

as was required. /I 
CoRollary 6.8. With $A$ as in Theorem 6.7, an element $q \in A$ fulfils the equality $q^{2}=-e$ if and only if

(a) $\|q\|=1$

(b) $q$ is orthogonal to $e$.

Proof. If $q^{2}=-e$ then, by (1) and (2), $(e, q)=0$ and $\|q\|=1$. Now let us suppose that the conditions (a) and (b) hold. Then we obtain

$$
\|e+\gamma q\|=\left(1+\gamma^{2}\right)^{\frac{1}{2}}
$$

and consequently

$$
\begin{gathered}
\tau(e, q)=\lim _{\gamma \rightarrow 0+}(\|e+\gamma q\|-1) / \gamma=0, \\
\tau(e,-q)=\lim _{\gamma \rightarrow 0+}(\|e+\gamma(-q)\|-1) / \gamma=0 .
\end{gathered}
$$

Hence, as it was shown in the proof of Theorem 6.4, the element $q$ fulfils the equality

$$
q^{2}=-\mu e, \quad \mu>0 .
$$

Therefore $q=\mu^{\frac{1}{2}} i$, where $i^{2}=-e$ and $\|i\|=1$. But $\|q\|=1$, which implies $\mu=1, q^{2}=-e$.

Definition. A normed space $\mathfrak{X}$ is said to be metrically homogeneous if for any pair of vectors $x_{1}, x_{2}$ with $\left\|x_{1}\right\|=\left\|x_{2}\right\|=1$ there exists a linear transformation $T$ of $\mathfrak{X}$ preserving the unit sphere $S=\{x \mid\|x\|=1\}$ such that $T\left(x_{1}\right)=x_{2}$.

THEOREM 6.9. Let $A$ be a real normed power-associative algebra containing the unit element e. If $A$ is a complete separable metrically homogeneous space, then $A$ is a (real) Hilbert space.

PROOF. It follows from our assumptions that there exists at least one vector $r$ with $\|r\|=1$ which is a regular point of a convex functional $p(x)=\|x\|$. (See e.g. [1], V.9.8). Let $T$ be the linear transformation preserving the unit sphere for which $T e=r$. Then for an arbitrary vector $y \in A$ we obtain

$$
\begin{aligned}
\tau(e, y) & =\lim _{\gamma \rightarrow 0+}(\|e+\gamma y\|-\|e\|) / \gamma \\
& =\lim _{\gamma \rightarrow 0+}(\|T(e+\gamma y)\|-\|T(e)\|) / \gamma \\
& =\lim _{\gamma \rightarrow 0+}(\|r+\gamma T(y)\|-\|\gamma\|) / \gamma=\tau(r, T(y)) .
\end{aligned}
$$

In the same way we verify that

$$
\tau(e,-y)=\tau(r,-T(y))
$$


But $-\tau(r,-T(y))=\tau(r, T(y))$ since $r$ is a regular point of $p(x)=\|x\|$. Therefore, $-\tau(e,-y)=\tau(e, y)$ for every $y \in A$, i.e. the unit element $e$ is a regular point of $p(x)=\|x\|$. Now our assertion follows from Theorem 6.7. $\|$

THEOREM 6.10. Each regular one-sided alternative real normed algebra is isometrically isomorphic with one of the following: the real field, the complex field, the quaternion algebra or the Cayley algebra.

This theorem follows from [8], Theorem 2 since, by Theorem 6.4, every algebra in question is algebraic.

We conclude with an example of a regular power-associative algebra.

EXAmpLE. Let $A$ be a real Hilbert space with an orthonormal basis $e_{0}, e_{1}, \cdots$. We define the multiplication of two elements

as follows

$$
x=\sum_{k=0}^{\infty} \xi_{k} e_{k} \text { and } y=\sum_{k=0}^{\infty} \eta_{k} e_{k}
$$

$$
x y=\left(\xi_{0} \eta_{0}-\sum_{k=1}^{\infty} \xi_{k} \eta_{k}\right) e_{0}+\sum_{k=1}^{\infty}\left(\xi_{0} \eta_{k}+\eta_{0} \xi_{k}\right) e_{k} .
$$

Let us write

$$
x_{n}=\sum_{k=0}^{n} \xi_{k} e_{k}, \quad y_{n}=\sum_{k=0}^{n} \eta_{k} e_{k} .
$$

We have then

$$
\begin{aligned}
\left\|x_{n} y_{n}\right\|^{2} & =\left(\xi_{0} \eta_{0}-\sum_{k=1}^{n} \xi_{k} \eta_{k}\right)^{2}+\sum_{k=1}^{n}\left(\xi_{0} \eta_{k}+\eta_{0} \xi_{k}\right)^{2} \\
& =\xi_{0}^{2} \eta_{0}^{2}+\left(\sum_{k=1}^{n} \xi_{k} \eta_{k}\right)^{2}+\xi_{0}^{2} \sum_{k=1}^{n} \eta_{k}^{2}+\eta_{0}^{2} \sum_{k=1}^{n} \xi_{k}^{2} \\
& \leqq \xi_{0}^{2} \sum_{k=0}^{n} \eta_{k}^{2}+\eta_{0}^{2} \sum_{k=1}^{n} \xi_{k}^{2}+\sum_{k=1}^{n} \xi_{k}^{2} \cdot \sum_{k=1}^{n} \eta_{k}^{2} \\
& =\sum_{k=0}^{n} \xi_{0}^{2} \cdot \sum_{k=0}^{n} \eta_{0}^{2}=\left\|x_{n}\right\|^{2} \cdot\left\|y_{n}\right\|^{2} .
\end{aligned}
$$

Since $\left\|x_{n}\right\|$ and $\left\|y_{n}\right\|$ do not decrease as $n \rightarrow \infty$, the last inequality yields

$$
\left\|x_{n} y_{n}\right\| \leqq\|x\| \cdot\|y\| \cdot
$$

This inequality is valid for every positive integer $n$ and therefore the element $x y \in A$ and

$$
\|x y\| \leqq\|x\| \cdot\|y\| .
$$

Now it is easy to verify that

(a) $e_{0}$ is the unit element of our algebra $A$,

(b) $A$ is regular and power-associative. 


\section{References}

[1] Dunford, N. and Schwartz, J. T. Linear operators, Part I, New York (1964).

[2] Ingelstam, L., "Non-associative normed algebras and Hurwitz' problem." Arkiv För Matematik 5 (1964), $231-238$.

[3] Ingelstam, L., "Real Banach algebras". Arkiv För Matematik 5 (1964), 239-270.

[4] Jordan, P. and Neumann, J. von, "On inner products in linear metric spaces", Ann. of Math. (2), 36 (1935), 719-723.

[5] Naimark, M. A., Normed rings, Groningen (1964).

[6] Natanson, I. P., Theory of funtions of a real variable, Vol. 1, New York (1955).

[7] Rickart, C. E., General theory of Banach algebras, Princeton (1960).

[8] Strzelecki, E., "Metric properties of normed algebras", Studia Math. 23 (1963), 41-51.

[9] Urbanik, K. and Wright, F. B., "Absolute-valued algebras", Proc. of the Amer. Math. Soc. 11, (1960), 861-866.

\section{Monash University}

Melbourne 